\title{
The thought processes behind instrument development
}

\author{
Don Lalonde MD FRCSC MSc BSc
}

$\mathrm{T}_{\mathrm{n}}^{\mathrm{h}}$ he following is a short description of the thought processes behind instrument development. Let's start by pretending that we have a room with eight interested surgeons and one good illustrator. They are assigned to develop a better bone clamp for open reduction of transverse fractures of the metacarpal, and they must end up with working drawings within 8 hours.

The first step is to believe that the current tools are not doing the job effectively. When there is adequate exposure of the anatomy, and a good understanding of the surgical problem, yet the surgeon struggles to get the tissues to do what he or she would like them to do with the current instrumentation, it is time to develop a new tool.

The second, and perhaps most important, step is to believe, assume or pretend that the new instrument is possible. The combined egos of the eight surgeons in that room would easily drive that fantasy into a reality within a short time frame. For single surgeons working at it on their own, however, there must be a desire to end the struggle with current tools that forces their brains to continue working on a new tool until they develop something that works.

The third step is to list the features that would be present in the ideal instrument for the job. In the case of the transverse metacarpal fracture, our eight surgeons would probably agree that the ideal bone clamp should:

- fit easily around the bone without bowstringing the tendons;

- allow room to see the bone so that plates, screws or $\mathrm{K}$ wires could be easily applied;

- hold the fracture solidly in anatomical reduction without assistance from the surgeon; and

- dynamically compress the fragments of bone.

The fourth step is to look at the components of the tool that currently work well. Bone clamps with sharp points (like towel clips) (Figure 1, top left) work well because they
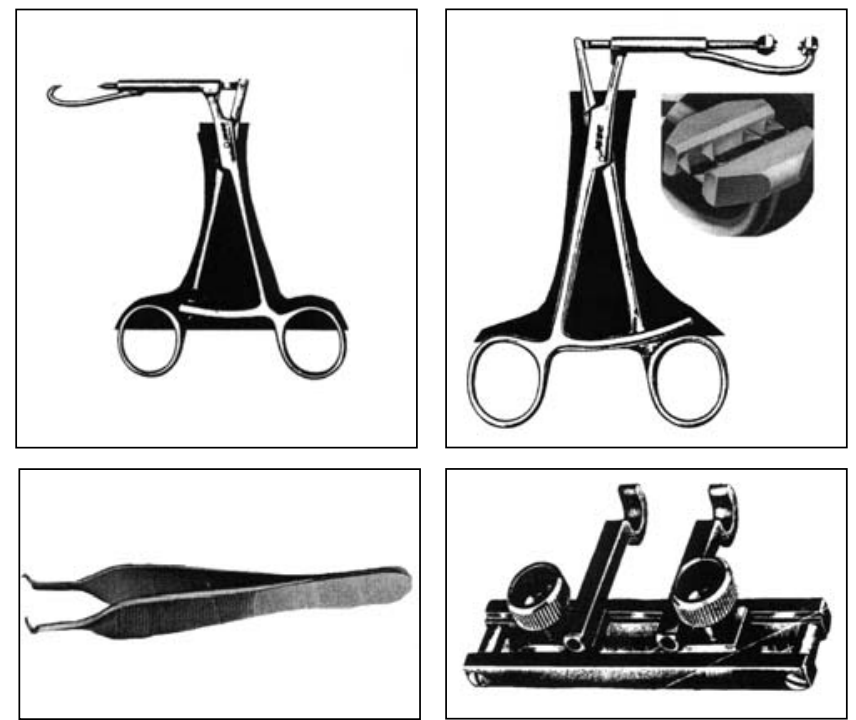

Figure 1) Top left Oblique fracture bone clamp. Top right Transverse fracture bone clamp. Bottom left Skin hook forceps. Bottom right Tendon approximator

do not let go of the bone. Dynamic compression plates work well by forcing the bone ends together as the screws bite into the bone and slide medially in the plate.

The fifth step is the fun part. This is where the designer figures out how to combine the effective components that have already been developed with those that still need to be developed. How do we get sharp points to enter the bone and drive the bone ends together at the same time? This process took years of thought before the final answer came about (Figure 1, top right). The most important part of this step is to believe that the new tool is possible and to be persistent in trying to find the answer to creating it.

Another way to develop ideas for new instruments is to imitate nature. The airplane is an excellent example of this type of thinking. Skin hook forceps (Figure 1, bottom left) are an imitation of the insect-like pincers of the earwig or of 
the biting red ants that the author used to watch as a child in northern Ontario.

A third way to develop new instruments is to take an existing tool used for a given purpose and modify it for another purpose. This approach was used in the development of the tendon approximator (Figure 1, bottom right). Vessel approximators already existed and use the concept of putting vessel clamps on arms that slide on a fixed bar. Applying the same concept to needles for skewering a tendon was really very simple.

The one thing that drives this type of thinking over and over again is the thought process that occurs while a surgical procedure is performed in which there is a struggle.

This isn't easy. Surgery should be easy if you understand the anatomy and what you are trying to do. There has got to be a better tool to help make this procedure easy. What would the ideal tool do here and what would it look like?
For many reasons, envisioning the tool is actually the easy part of instrument development. The difficulty is in building prototypes, convincing people in the industry that it is worth investing the large sums of money that are required to produce the tool and, finally, overcoming all the hurdles of successful marketing. Roughly, only $1 \%$ of surgical instruments that reach the successful prototype stage actually go on to successful marketing and widespread surgical use.

This author believes that the year 2002 sees surgical technology in its infancy. From a practical point of view, our planet has seen only four generations of surgeons. Why? Because the average working lifespan of a surgeon is only about 30 years, and because it is only about 120 years ago that surgical technology was really allowed to begin serious development with the advent of general anesthesia and sterile techniques. There is plenty of room for improvement in our operating rooms.

Let's get at it. 